\title{
Adaptação transcultural da Escala de Atitudes Antiobesidade para o português do Brasil
}

\author{
Transcultural adaptation of the Antifat Attitudes Test \\ to Brazilian Portuguese
}

Angélica Almeida Obara ${ }^{1}$

Marle dos Santos Alvarenga ${ }^{1}$

${ }^{1}$ Faculdade de Saúde Pública, Universidade de São Paulo. Av. Dr. Arnaldo 715, Cerqueira César. 03178 200 São Paulo SP Brasil. ge_angelica@hotmail.com
Abstract Obese individuals are often blamed for their own condition and the targets of discrimination and prejudice. The scope of this study is to describe the cross-cultural adaptation to Brazilian Portuguese and the validation of the Antifat Attitudes Test - specifically developed for evaluation of negative attitudes toward the obese individual. The scale has 34 statements distributed in three subscales - Social/Character Disparagement (15 items), Physical/Romantic Unattractiveness (10 items) and Weight Control/Blame (9 items). The method involved the translation of the scale; evaluation of the conceptual, operational and item equivalence; evaluation of the semantic equivalence using the paired t test, the Pearson correlation coefficient and the intraclass correlation coefficient (ICC); internal consistency evaluation (Cronbach's alpha) and test-retest reliability (ICC) and Confirmatory Factor Analysis - after application in 340 college students in the area of health. The results showed good global internal consistency and reliability ( $\alpha$ 0.85; CCI 0.83), and factor analysis showed that the original subscales can be kept in the adaptation, and therefore the scale adapted to the Brazilian-Portuguese version is valid and useful in studies to explore negative attitudes toward obese individuals.

Key words Scales, Validation studies, Obesity
Resumo Indivíduos obesos são muitas vezes considerados culpados por sua condição e alvos de discriminação e preconceito. O objetivo deste estudo é descrever a adaptação transcultural para o português do Brasil e validação do Antifat Attitudes Test - desenvolvido especificamente para a avaliação de atitudes negativas para com o indivíduo obeso. A escala possui 34 itens distribuídos em três subescalas nomeadas de "depreciação social e do caráter" (15 itens), "não atratividade física $e$ romântica" (10 itens) e "controle do peso e culpa" (9 itens). O método envolveu a tradução da escala; avaliação da equivalência conceitual, operacional $e$ de itens; avaliação da equivalência semântica por meio do teste t pareado, do coeficiente de correlação de Pearson e coeficiente de correlação intraclasse (CCI); avaliação da consistência interna (Alpha de Cronbach), confiabilidade teste-reteste (CCI) e Análise Fatorial Confirmatória - após aplicação em 340 universitários da área da saúde. Os resultados apontaram boa consistência interna e confiabilidade para a escala global ( $\alpha 0,85$; CCI 0,83), e a análise fatorial demonstrou que as subescalas originais podem ser mantidas na adaptação, sendo, portanto, a escala adaptada para o português do Brasil, válida e útil em estudos para explorar atitudes negativas para com os indivíduos obesos.

Palavras-chave Escalas, Estudos de validação, Obesidade 


\section{Introdução}

A obesidade constitui um grave problema de saúde pública mundial e está relacionada a efeitos metabólicos adversos e maior risco de algumas doenças ${ }^{1}$. Consequências psicológicas e sociais também estão relacionadas à obesidade, e há hoje uma tendência à globalização do seu estigma² ${ }^{2}$.

Apesar da relevância dos fatores ambientais na sua etiologia ${ }^{3,4}$, os indivíduos obesos são considerados culpados por sua condição e alvos frequentes de discriminação e preconceito em cenários como trabalho, escola, mídia e serviços de saúde ${ }^{5-7}$. Mesmo aqueles envolvidos no tratamento da obesidade (médicos, nutricionistas, enfermeiros e psicólogos) demonstraram preconceito e atitudes negativas direcionados à obesidade e aos indivíduos obesos ${ }^{3,8-12}$ - e o mesmo foi verificado entre estudantes da área da saúde ${ }^{11,13-15}$.

O estigma é definido como um atributo negativo constituído a partir da discrepância entre a identidade social virtual (características previamente atribuídas por um outrem ao entrar em contato com um indivíduo) e a identidade social real. Por meio de relações entre determinados atributos e estereótipos, o sujeito percebido como diferente sofre discriminação, mesmo que de maneira inconsciente, o que ocasiona a diminuição de suas chances na vida ${ }^{16}$. Preconceito é uma atitude hostil ou aversiva direcionada àqueles que possuem qualidades consideradas censuráveis socialmente, posicionando de forma desvantajosa em função de características que diferenciem a pessoa ou grupo em um determinado contexto social ${ }^{17}$. O preconceito em relação à obesidade engloba atitudes, crenças e comportamentos contrários ao indivíduo percebido como obeso ou "gordo"18. A discriminação é considerada a manifestação comportamental do preconceito. Nos Estados Unidos a discriminação relacionada ao peso, referida por uma amostra representativa de adultos, apresentou um aumento de $66 \% \mathrm{du}-$ rante a última década ${ }^{19}$.

Por sua vez, atitude é um construto com três componentes: $\mathrm{o}$ afetivo que se refere a sentimentos, humor e emoções; o cognitivo que se refere a crenças e conhecimentos; e o componente volitivo, de vontade, se refere à intenção comportamental em relação ao objeto ${ }^{20}$. Assim, o interesse em estudar as atitudes está na compreensão e na predição de comportamentos ${ }^{21}$.

No contexto da obesidade, sabe-se que o estigma e o preconceito não contribuem para $o$ tratamento e redução dos índices de obesidade; e ao contrário, podem trazer diversas implicações negativas para a saúde do indivíduo obeso ${ }^{7}$.
O preconceito pode ser expresso de maneira explícita, por exemplo, quando um indivíduo responde conscientemente a um questionário; ou implícita, por exemplo, quando revela aspectos que o indivíduo reluta ou é incapaz de reportar de forma consciente ${ }^{22}$. A maioria dos estudos que investigam a presença de preconceito e atitudes negativas utiliza métodos explícitos, por meio de escalas ${ }^{23}$. Diversas escalas foram desenvolvidas com foco nesta temática, com objetivos e populações variadas. As primeiras escalas datam da década de 1990 e avaliam atitudes em relação aos indivíduos obesos e crenças em relação ao controle da obesidade por parte do mesmo ${ }^{24}$. Há escalas específicas elaboradas para aplicação em profissionais de saúde - com questões focadas em suas atitudes em relação à obesidade e no contexto de interação com pacientes obesos ${ }^{25,26}$. Outras não avaliam atitudes e sim estereótipos relacionados ao peso corporal ou ao obeso ${ }^{27,28}$. Apenas três trabalhos exploram esta temática entre profissionais de saúde no Brasil e sem uso de instrumentos padronizados ${ }^{29-31}$. Nenhuma das escalas específicas sobre esta temática tem versão validada para o português do Brasil.

Dentre as escalas que avaliam as atitudes em relação à obesidade, a Antifat Attitudes Test AFAT $^{32}$ foi desenvolvida e avaliada com estudantes universitários americanos e possui itens que abordam três dimensões das atitudes em relação à obesidade e aos obesos: a "depreciação social e do caráter", a "não atratividade física e romântica" (uma dimensão não explorada anteriormente em outros trabalhos), e o "controle do peso e culpa". Os autores destacam ainda que comparada a outras escalas que avaliam o construto, a AFAT avalia estas atitudes negativas sem confundi-las com desejo de aceitação social, e sem contaminação com crenças que possam refletir possíveis riscos à saúde ou vitimização social. A AFAT em sua primeira versão possuía 47 itens; e em avaliação posterior com estudantes universitários americanos, 34 itens atingiram os valores mínimos na análise fatorial de componentes principais e foram incluídos em uma das três subescalas ${ }^{32}$. Esta segunda versão apresentou características psicométricas satisfatórias e consistência interna adequada ( $\alpha$ Cronbach $=0,95$ ).

$\mathrm{O}$ instrumento tem interessantes possibilidades de aplicação, e uma vez que foi desenvolvido e validado para língua inglesa, é necessária sua adaptação para uso no cenário brasileiro. O presente estudo tem, portanto, o objetivo de apresentar o processo de adaptação transcultural da escala Antifat Attitudes Test para o português denominada de Escala de Atitudes Anti-obesida- 
de - e suas propriedades psicométricas para uso no Brasil.

\section{Métodos}

As etapas do processo de adaptação transcultural seguiram as recomendações gerais vigentes ${ }^{33}$ e envolveram a análise das equivalências conceitual, de itens, semântica, operacional e de mensuração.

Na presente adaptação optou-se por utilizar a segunda versão da AFAT com os 34 itens que atingiram os valores mínimos na análise fatorial distribuídas em três subescalas, nomeadas pelos autores como 1. "depreciação social e do caráter" (15 itens que avaliam a atribuição de características pessoais socialmente indesejáveis e desprezo social em relação aos obesos), 2. "não atratividade física e romântica” (10 itens que refletem percepções de que os obesos são desajeitados, não atraentes e inaceitáveis como parceiros românticos) e 3. "controle do peso e culpa" (9 itens que avaliam a crença quanto à responsabilidade dos obesos por seu excesso de peso). As respostas são dadas em escala do tipo Likert com cinco opções (variando entre discordo totalmente e concordo totalmente - pontuações de 1 a 5 , respectivamente), na qual maiores escores refletem atitudes mais negativas em relação à obesidade e aos indivíduos obesos.

O escore total da escala é calculado, conforme orientação dos autores do instrumento original, a partir do somatório das pontuações de cada afirmação dividido pelo número de itens da escala, ou seja, 34. Os escores das subescalas são calculados da mesma forma, por meio da soma das pontuações obtidas em cada item dividida pelo número de itens de cada subescala (ou seja, 15, 10 ou 9). Dessa forma, a menor pontuação possível para a escala global e para as subescalas é de 1 ponto e a máxima de 5 pontos.

\section{Etapas da adaptação transcultural}

A avaliação da equivalência conceitual e de itens consiste na exploração do construto de interesse, da relevância e pertinência dos domínios da escala no país de origem e no contexto sociocultural em que será utilizado. Esta etapa, da mesma forma como em outros trabalhos de adaptação, foi realizada por meio da discussão do instrumento com experts (15 nutricionistas com atuação clínica em obesidade e transtornos alimentares). Antes da equivalência semântica
- descrita a seguir - os experts receberam a escala original por correio eletrônico e avaliaram a mesma (seus itens, o texto e o uso em estudos internacionais), e responderam sobre a pertinência das questões para a avaliação de atitudes "antiobesidade" no cenário do Brasil. Eles deveriam responder se consideravam a mesma adequada, e enviarem comentários sobre possibilidade de uso no Brasil e considerações gerais. As respostas foram discutidas com os 15 profissionais por correio eletrônico e telefone. Todos consideraram a escala adequada para adaptação no nosso contexto e relevante para o estudo da presença de atitudes negativas em relação à obesidade.

Após “aprovação" dos experts, os autores da AFAT foram contatados para anuência quanto ao processo de adaptação transcultural e autorizaram o mesmo.

A avaliação de equivalência semântica se relaciona à capacidade de transferir o significado de conceitos contidos no instrumento original para a versão adaptada. A tradução da escala foi realizada por dois bilíngues (com fluência em português e inglês) de forma independente, e em seguida, as duas versões foram extensamente discutidas para alcançar uma versão única que apresentasse melhor equivalência conceitual e adequação para o público-alvo (jovens universitários). A versão única foi apresentada a um grupo de 15 nutricionistas (experts) com experiência em obesidade, transtornos alimentares, atitudes alimentares - e alguns deles com experiência em adaptação transcultural de instrumentos - para verificar o grau de compreensão das afirmações (opções de resposta: 1 = não entendi nada/ 2 = entendi só um pouco/ 3 = entendi mais ou menos/ 4 = entendi quase tudo, mas tive algumas dúvidas/ 5 = entendi perfeitamente e não tenho dúvidas) e discussão da tradução de termos que geraram dúvidas após a tradução inicial. Um linguista profissional, graduado em letras, com especialização em língua inglesa também foi consultado para solucionar dúvidas em relação a termos levantados. As sugestões de modificações e correções realizadas pelos nutricionistas foram consideradas para a elaboração da versão traduzida final.

Ademais, para ampliação da avaliação da capacidade dos conceitos do instrumento original em serem transferidos para a versão adaptada, como etapa complementar da equivalência semântica, 13 adultos jovens bilíngues foram convidados a responder a versão traduzida e a versão original em inglês da escala de forma on-line. Desta forma, buscou-se avaliar se os itens nas 
versões em inglês e português foram entendidos da mesma forma. Os bilíngues foram divididos aleatoriamente em dois grupos: um deles respondeu primeiro a versão traduzida e após intervalo de no mínimo cinco dias, respondeu a versão em inglês; enquanto o outro grupo recebeu a primeira versão em inglês e a versão em português após os cinco dias. Para a comparação das respostas em português e em inglês, foram atribuídos escores às respostas oferecidas em ambos os questionários: 1 = definitely disagree ou discordo totalmente $/ 2$ mostly disagree ou discordo da maior parte/ $3=$ neither agree nor disagree ou nem concordo nem discordo $/ 4=$ mostly agree ou concordo com a maior parte/ $5=$ definitely agree ou concordo totalmente. Foram consideradas incongruências importantes as respostas que apresentaram diferença maior do que dois pontos entre a assinalada em português e a em inglês; e as afirmações que obtiveram baixa correlação na análise estatística foram novamente discutidas com os nutricionistas (experts) para novas alterações.

Como parte final da equivalência semântica, a retrotradução da escala final adaptada foi realizada por outro bilíngue, de forma independente (não envolvido na tradução), considerando-se qualquer necessidade de alteração de linguagem feita pelos experts e bilíngues.

A análise da equivalência operacional se refere à avaliação do veículo e formato das questões e instruções, do cenário de administração, do modo de aplicação e de categorização e consistiu na aplicação da versão final adaptada em português para 37 estudantes ( 17 de graduação em nutrição e 20 estudantes de pós-graduação em Saúde Pública). Estes deveriam responder a escala, com anotação do tempo de preenchimento total, e após o mesmo, responderem sobre a clareza das afirmações e apontarem qualquer dificuldade no preenchimento da escala, ou dúvidas e considerações sobre os itens da mesma.

Por fim, a análise de mensuração, compreende a investigação das propriedades psicométricas do instrumento adaptado; para os quais foram testadas a consistência interna, a confiabilidade teste-reteste e a análise fatorial confirmatória. Participaram desta etapa 340 estudantes de graduação em enfermagem e fisioterapia que responderam a versão em português da AFAT em sala de aula. Aproximadamente 30 dias depois e após sorteio, aqueles que disponibilizaram seu endereço eletrônico (e-mail) receberam novo contato para responder novamente a escala para análise da confiabilidade teste-reteste.

\section{Considerações éticas}

O estudo foi realizado de acordo com os princípios éticos envolvidos na pesquisa em seres humanos e aprovado pelo Comitê de Ética da Faculdade de Saúde Pública da Universidade de São Paulo. Os participantes das etapas de equivalência operacional e mensuração assinaram termo de consentimento livre e esclarecido.

\section{Análise estatística}

A análise estatística foi realizada utilizando o software Statistical Package for Social Sciences (SPSS) 21.0. Para todas as análises foi adotado um nível de significância de $p \leq 0,05$.

A avaliação dos experts $(\mathrm{N}=15)$ na equivalência semântica foi avaliada por frequência de resposta. Na avaliação com os jovens bilíngues (N = 13), a equivalência semântica foi avaliada pelo teste Wilcoxon (equivalente ao teste $t$ pareado para dados não paramétricos), pelo coeficiente de correlação de Pearson, que aponta a correlação entre postos e linear entre as duas aplicações, e pelo coeficiente de correlação intraclasse (CCI), que aponta a confiabilidade entre as medidas.

A consistência interna da escala adaptada foi avaliada por meio do Alpha de Cronbach - assim como no desenvolvimento da AFAT original ${ }^{32}$; e a confiabilidade teste-reteste pelo coeficiente de correlação intraclasse (CCI) - para verificar como as duas medidas (teste e reteste) dos escores da AFAT se comportaram com o intervalo de tempo.

A análise fatorial confirmatória (AFC) foi realizada para avaliar se a mesma estrutura fatorial da escala original (3 fatores e os itens que os compõe) poderia ser mantida na escala adaptada. Desta forma, a AFC foi conduzida por meio do programa R 3.2.1 com o pacote Lavaan utilizando o método de estimação de mínimos quadrados ponderado robusto (não depende da normalidade das variáveis) e correlação policórica (para variáveis ordinais). O Comparative Fit Index (CFI) e Tucker-Lewis Index (TLI) foram os índices utilizados para avaliação do modelo (bem ajustados quando acima de 0,9 ); bem como o Root Mean Square Error of Aproximation (RMSEA) - bom ajuste quando abaixo de 0,08.

\section{Resultados}

As versões da AFAT estão apresentadas no Quadro 1. 
Quadro 1. Versões do Antifat Attitudes Test (AFAT).

\begin{tabular}{|c|c|c|c|c|}
\hline $\mathrm{n}$ & Versão original & Versão traduzida 1 & Versão adaptada final & Versão retrotraduzida \\
\hline 1 & $\begin{array}{l}\text { There's no excuse for } \\
\text { being fat }\end{array}$ & $\begin{array}{l}\text { Não há desculpa para ser } \\
\text { gordo }\end{array}$ & $\begin{array}{l}\text { Não há desculpa para } \\
\text { ser gordo }\end{array}$ & $\begin{array}{l}\text { There is no excuse to } \\
\text { be fat }\end{array}$ \\
\hline 2 & $\begin{array}{l}\text { If I were single, I would } \\
\text { date a fat person }\end{array}$ & $\begin{array}{l}\text { Se eu fosse solteiro (a), eu } \\
\text { namoraria uma pessoa } \\
\text { gorda }\end{array}$ & $\begin{array}{l}\text { Se eu fosse solteiro } \\
\text { (a), eu namoraria uma } \\
\text { pessoa gorda }\end{array}$ & $\begin{array}{l}\text { If I were single, I would } \\
\text { date a fat person }\end{array}$ \\
\hline 3 & $\begin{array}{l}\text { Most fat people buy too } \\
\text { much junk food }\end{array}$ & $\begin{array}{l}\text { A maioria dos gordos } \\
\text { compra muita besteira } \\
\text { (“junk food”) }\end{array}$ & $\begin{array}{l}\text { A maioria dos gordos } \\
\text { compra muita besteira } \\
\text { (“junk food”) }\end{array}$ & $\begin{array}{l}\text { The most part of fat } \\
\text { people buys a lot of junk } \\
\text { food }\end{array}$ \\
\hline 4 & $\begin{array}{l}\text { Fat people are physically } \\
\text { unattractive }\end{array}$ & $\begin{array}{l}\text { Pessoas gordas não são } \\
\text { atraentes }\end{array}$ & $\begin{array}{l}\text { Pessoas gordas não são } \\
\text { atraentes }\end{array}$ & $\begin{array}{l}\text { Fat people are not } \\
\text { attractive }\end{array}$ \\
\hline 5 & $\begin{array}{l}\text { Fat people shouldn't } \\
\text { wear revealing clothing } \\
\text { in public }\end{array}$ & $\begin{array}{l}\text { Pessoas gordas não } \\
\text { deveriam usar roupas que } \\
\text { mostram demais o corpo }\end{array}$ & $\begin{array}{l}\text { Pessoas gordas não } \\
\text { deveriam usar em } \\
\text { público roupas que } \\
\text { mostram demais o } \\
\text { corpo }\end{array}$ & $\begin{array}{l}\text { Fat people should not } \\
\text { wear clothes that show } \\
\text { so much skin in public } \\
\text { settings }\end{array}$ \\
\hline 6 & $\begin{array}{l}\text { If fat people don't get } \\
\text { hired, it's their own fault }\end{array}$ & $\begin{array}{l}\text { Se pessoas gordas não } \\
\text { conseguem emprego, é } \\
\text { culpa delas mesmas }\end{array}$ & $\begin{array}{l}\text { Se pessoas gordas não } \\
\text { são contratadas para } \\
\text { um emprego, a culpa é } \\
\text { delas mesmas }\end{array}$ & $\begin{array}{l}\text { If fat people are not hired } \\
\text { for a job, it is their fault }\end{array}$ \\
\hline 7 & $\begin{array}{l}\text { Fat people don't care } \\
\text { about anything except } \\
\text { eating }\end{array}$ & $\begin{array}{l}\text { Pessoas gordas não se } \\
\text { importam com nada além } \\
\text { de comer }\end{array}$ & $\begin{array}{l}\text { Pessoas gordas não se } \\
\text { importam com nada } \\
\text { além de comer }\end{array}$ & $\begin{array}{l}\text { Fat people do not care } \\
\text { with anything besides } \\
\text { eating }\end{array}$ \\
\hline 8 & $\begin{array}{l}\text { I'd lose respect for a } \\
\text { friend who started } \\
\text { getting fat }\end{array}$ & $\begin{array}{l}\text { Eu perderia respeito por } \\
\text { um (a) amigo (a) que } \\
\text { começasse a engordar }\end{array}$ & $\begin{array}{l}\text { Eu perderia o respeito } \\
\text { por um (a) amigo (a) } \\
\text { que começasse a ficar } \\
\text { gordo (a) }\end{array}$ & $\begin{array}{l}\text { I would lose respect by a } \\
\text { friend who would start } \\
\text { putting on weight }\end{array}$ \\
\hline 9 & $\begin{array}{l}\text { Most fat people are } \\
\text { boring }\end{array}$ & $\begin{array}{l}\text { A maioria das pessoas } \\
\text { gordas é chata }\end{array}$ & $\begin{array}{l}\text { A maioria das pessoas } \\
\text { gordas é chata }\end{array}$ & $\begin{array}{l}\text { The most part of fat } \\
\text { people is boring }\end{array}$ \\
\hline 10 & $\begin{array}{l}\text { I can't believe someone } \\
\text { of average weight would } \\
\text { marry a fat person }\end{array}$ & $\begin{array}{l}\text { Eu não acredito que uma } \\
\text { pessoa de peso normal se } \\
\text { casaria com uma pessoa } \\
\text { gorda }\end{array}$ & $\begin{array}{l}\text { Eu não acredito que } \\
\text { uma pessoa de peso } \\
\text { normal se casaria com } \\
\text { uma pessoa gorda }\end{array}$ & $\begin{array}{l}\text { I don't believe a normal } \\
\text { weight person would } \\
\text { marry a fat person }\end{array}$ \\
\hline 11 & $\begin{array}{l}\text { Society is too tolerant of } \\
\text { fat people }\end{array}$ & $\begin{array}{l}\text { A sociedade é muito } \\
\text { tolerante com os gordos }\end{array}$ & $\begin{array}{l}\text { A sociedade é muito } \\
\text { tolerante com as } \\
\text { pessoas gordas }\end{array}$ & $\begin{array}{l}\text { Society is very tolerant } \\
\text { with fat people }\end{array}$ \\
\hline 12 & $\begin{array}{l}\text { When fat people } \\
\text { exercise, they look } \\
\text { ridiculous }\end{array}$ & $\begin{array}{l}\text { Quando pessoas gordas } \\
\text { fazem exercício, elas } \\
\text { parecem ridículas }\end{array}$ & $\begin{array}{l}\text { Quando pessoas gordas } \\
\text { fazem exercício, elas } \\
\text { parecem ridículas }\end{array}$ & $\begin{array}{l}\text { When fat people do } \\
\text { exercise, they seem } \\
\text { ridiculous }\end{array}$ \\
\hline 13 & Most fat people are lazy & $\begin{array}{l}\text { A maioria dos gordos é } \\
\text { preguiçosa }\end{array}$ & $\begin{array}{l}\text { A maioria das pessoas } \\
\text { gordas é preguiçosa }\end{array}$ & $\begin{array}{l}\text { The most part of fat } \\
\text { people is lazy }\end{array}$ \\
\hline 14 & $\begin{array}{l}\text { Fat people are Just as } \\
\text { competent in their work } \\
\text { as anyone }\end{array}$ & $\begin{array}{l}\text { As pessoas gordas são } \\
\text { tão competentes no seu } \\
\text { trabalho quanto qualquer } \\
\text { um }\end{array}$ & $\begin{array}{l}\text { As pessoas gordas são } \\
\text { tão competentes no } \\
\text { seu trabalho quanto } \\
\text { qualquer um }\end{array}$ & $\begin{array}{l}\text { Fat people are as } \\
\text { competent at work as } \\
\text { anyone is }\end{array}$ \\
\hline 15 & $\begin{array}{l}\text { If fat people really } \\
\text { wanted to lose weight, } \\
\text { they could }\end{array}$ & $\begin{array}{l}\text { Se as pessoas gordas } \\
\text { realmente quisessem } \\
\text { emagrecer, elas } \\
\text { conseguiriam }\end{array}$ & $\begin{array}{l}\text { Se as pessoas gordas } \\
\text { realmente quisessem } \\
\text { emagrecer, elas } \\
\text { conseguiriam }\end{array}$ & $\begin{array}{l}\text { If fat people really } \\
\text { wanted to lose weight, } \\
\text { they would succeed }\end{array}$ \\
\hline 16 & Being fat is sinful & Ser gordo é pecado & Ser gordo é pecado & To be fat is a sin \\
\hline 17 & $\begin{array}{l}\text { It's disgusting to see fat } \\
\text { people eating }\end{array}$ & $\begin{array}{l}\text { É nojento ver pessoas } \\
\text { gordas comendo }\end{array}$ & $\begin{array}{l}\text { É nojento ver pessoas } \\
\text { gordas comendo }\end{array}$ & $\begin{array}{l}\text { It is disgusting to see fat } \\
\text { people eating }\end{array}$ \\
\hline 18 & $\begin{array}{l}\text { Fat people have no } \\
\text { willpower }\end{array}$ & $\begin{array}{l}\text { Pessoas gordas não têm } \\
\text { força de vontade }\end{array}$ & $\begin{array}{l}\text { Pessoas gordas não têm } \\
\text { força de vontade }\end{array}$ & $\begin{array}{l}\text { Fat people do not have } \\
\text { willpower }\end{array}$ \\
\hline
\end{tabular}


Quadro 1. continuação

\begin{tabular}{|c|c|c|c|c|}
\hline $\mathbf{n}$ & Versão original & Versão traduzida 1 & Versão adaptada final & Versão retrotraduzida \\
\hline 19 & $\begin{array}{l}\text { I prefer not to associate } \\
\text { with fat people }\end{array}$ & $\begin{array}{l}\text { Eu prefiro não me associar } \\
\text { a pessoas gordas }\end{array}$ & $\begin{array}{l}\text { Eu prefiro não me } \\
\text { relacionar com pessoas } \\
\text { gordas }\end{array}$ & $\begin{array}{l}\text { I prefer to avoid } \\
\text { relationship with fat } \\
\text { people }\end{array}$ \\
\hline 20 & $\begin{array}{l}\text { Most fat people are } \\
\text { moody and hard to get } \\
\text { along with }\end{array}$ & $\begin{array}{l}\text { A maioria das pessoas } \\
\text { gordas é temperamental e } \\
\text { difícil de lidar }\end{array}$ & $\begin{array}{l}\text { A maioria das pessoas } \\
\text { gordas é temperamental } \\
\text { e difícil de lidar }\end{array}$ & $\begin{array}{l}\text { The most part of people } \\
\text { is moody and difficult to } \\
\text { handle }\end{array}$ \\
\hline 21 & $\begin{array}{l}\text { If bad things happen to } \\
\text { fat people, they deserve } \\
\text { it }\end{array}$ & $\begin{array}{l}\text { Se coisas ruins acontecem } \\
\text { com pessoas gordas, elas } \\
\text { merecem }\end{array}$ & $\begin{array}{l}\text { Se coisas ruins } \\
\text { acontecem com pessoas } \\
\text { gordas, elas merecem }\end{array}$ & $\begin{array}{l}\text { If bad situations happen } \\
\text { to fat people, they } \\
\text { deserve it }\end{array}$ \\
\hline 22 & $\begin{array}{l}\text { Most fat people don't } \\
\text { keep their surroundings } \\
\text { neat and clear }\end{array}$ & $\begin{array}{l}\text { A maioria das pessoas } \\
\text { gordas não consegue } \\
\text { manter as coisas limpas e } \\
\text { organizadas } \\
\end{array}$ & $\begin{array}{l}\text { A maioria das pessoas } \\
\text { gordas não consegue } \\
\text { manter as coisas limpas } \\
\text { e organizadas }\end{array}$ & $\begin{array}{l}\text { The most part of fat } \\
\text { people cannot keep their } \\
\text { things tidy and organized }\end{array}$ \\
\hline 23 & $\begin{array}{l}\text { Society should respect } \\
\text { the rights of fat people }\end{array}$ & $\begin{array}{l}\text { A sociedade deveria } \\
\text { respeitar os direitos dos } \\
\text { gordos }\end{array}$ & $\begin{array}{l}\text { A sociedade deveria } \\
\text { respeitar os direitos das } \\
\text { pessoas gordas }\end{array}$ & $\begin{array}{l}\text { Society should respect fat } \\
\text { people rights }\end{array}$ \\
\hline 24 & $\begin{array}{l}\text { It's hard not to stare at } \\
\text { fat people because they } \\
\text { are so unattractive }\end{array}$ & $\begin{array}{l}\text { É difícil não encarar as } \\
\text { pessoas gordas porque elas } \\
\text { são pouco atrativas }\end{array}$ & $\begin{array}{l}\text { É difícil não encarar as } \\
\text { pessoas gordas porque } \\
\text { elas são pouco atraentes }\end{array}$ & $\begin{array}{l}\text { It is hard to face fat } \\
\text { people because they are } \\
\text { unattractive }\end{array}$ \\
\hline 25 & $\begin{array}{l}\text { The Idea that genetics } \\
\text { causes people to be fat is } \\
\text { Just an excuse }\end{array}$ & $\begin{array}{l}\text { A ideia que a genética } \\
\text { causa a obesidade é } \\
\text { simplesmente uma } \\
\text { desculpa }\end{array}$ & $\begin{array}{l}\text { A ideia que genética } \\
\text { causa obesidade é } \\
\text { simplesmente uma } \\
\text { desculpa }\end{array}$ & $\begin{array}{l}\text { The idea that genetics } \\
\text { causes obesity is simply } \\
\text { excuse }\end{array}$ \\
\hline 26 & $\begin{array}{l}\text { I would not want to } \\
\text { continue in a romantic } \\
\text { relationship if my } \\
\text { partner became fat }\end{array}$ & $\begin{array}{l}\text { Eu não continuaria num } \\
\text { relacionamento romântico } \\
\text { se meu (minha) parceiro } \\
\text { (a) começasse a engordar }\end{array}$ & $\begin{array}{l}\text { Eu não continuaria } \\
\text { num relacionamento } \\
\text { amoroso se meu } \\
\text { (minha) parceiro (a) se } \\
\text { tornasse gordo (a) }\end{array}$ & $\begin{array}{l}\text { I would not keep a } \\
\text { love relationship if my } \\
\text { partner became a fat } \\
\text { person }\end{array}$ \\
\hline 27 & $\begin{array}{l}\text { I don't understand } \\
\text { how someone could be } \\
\text { sexually attracted to a } \\
\text { fat person }\end{array}$ & $\begin{array}{l}\text { Eu não entendo como } \\
\text { alguém pode se sentir } \\
\text { sexualmente atraído por } \\
\text { uma pessoa gorda }\end{array}$ & $\begin{array}{l}\text { Eu não entendo como } \\
\text { alguém pode se sentir } \\
\text { sexualmente atraído por } \\
\text { uma pessoa gorda }\end{array}$ & $\begin{array}{l}\text { I do not understand how } \\
\text { a person can be sexually } \\
\text { attracted to a fat person }\end{array}$ \\
\hline 28 & $\begin{array}{l}\text { If fat people knew how } \\
\text { bad they looked, they } \\
\text { would lose weight }\end{array}$ & $\begin{array}{l}\text { Se as pessoas gordas } \\
\text { soubessem quão ruim é } \\
\text { sua aparência, elas iriam } \\
\text { emagrecer }\end{array}$ & $\begin{array}{l}\text { Se as pessoas gordas } \\
\text { soubessem quão ruim } \\
\text { é sua aparência, elas } \\
\text { emagreceriam }\end{array}$ & $\begin{array}{l}\text { If fat people knew how } \\
\text { bad their corporal image } \\
\text { is, they would lose weight }\end{array}$ \\
\hline 29 & $\begin{array}{l}\text { People who are fat } \\
\text { have as much physical } \\
\text { coordination as anyone }\end{array}$ & $\begin{array}{l}\text { Pessoas gordas têm tanta } \\
\text { coordenação motora } \\
\text { quanto qualquer um }\end{array}$ & $\begin{array}{l}\text { Pessoas gordas têm } \\
\text { tanta coordenação } \\
\text { motora quanto } \\
\text { qualquer outra } \\
\end{array}$ & $\begin{array}{l}\text { Fat people have motor } \\
\text { coordination as any } \\
\text { other person }\end{array}$ \\
\hline 30 & Fat people are unclean & Pessoas gordas são sujas & $\begin{array}{l}\text { Pessoas gordas não são } \\
\text { higiênicas }\end{array}$ & $\begin{array}{l}\text { Fat people are } \\
\text { unhygienic }\end{array}$ \\
\hline 31 & $\begin{array}{l}\text { Fat people should be } \\
\text { encouraged to accept } \\
\text { themselves the way they } \\
\text { are }\end{array}$ & $\begin{array}{l}\text { Pessoas gordas deveriam } \\
\text { ser encorajadas a se } \\
\text { aceitarem como são }\end{array}$ & $\begin{array}{l}\text { Pessoas gordas } \\
\text { deveriam ser } \\
\text { encorajadas a se } \\
\text { aceitarem como são }\end{array}$ & $\begin{array}{l}\text { Fat people should be } \\
\text { encouraged to accept } \\
\text { themselves as they are }\end{array}$ \\
\hline 32 & $\begin{array}{l}\text { Most fat people will } \\
\text { latch onto almost any } \\
\text { excuse for being fat }\end{array}$ & $\begin{array}{l}\text { A maioria das pessoas } \\
\text { gordas se prende a } \\
\text { qualquer desculpa para } \\
\text { estar gorda }\end{array}$ & $\begin{array}{l}\text { A maioria das pessoas } \\
\text { gordas se prende a } \\
\text { qualquer desculpa para } \\
\text { estar gorda }\end{array}$ & $\begin{array}{l}\text { The most part of fat } \\
\text { people uses excuses } \\
\text { about being fat }\end{array}$ \\
\hline 33 & $\begin{array}{l}\text { It's hard to take fat } \\
\text { people seriously }\end{array}$ & $\begin{array}{l}\text { É difícil levar uma pessoa } \\
\text { gorda a sério }\end{array}$ & $\begin{array}{l}\text { É difícil levar uma } \\
\text { pessoa gorda a sério }\end{array}$ & $\begin{array}{l}\text { It is difficult to take a fat } \\
\text { person seriously }\end{array}$ \\
\hline 34 & $\begin{array}{l}\text { Fat people do not } \\
\text { necessarily eat more } \\
\text { than other people }\end{array}$ & $\begin{array}{l}\text { Pessoas gordas não } \\
\text { necessariamente comem } \\
\text { mais que os outros }\end{array}$ & $\begin{array}{l}\text { Pessoas gordas não } \\
\text { necessariamente } \\
\text { comem mais que os } \\
\text { outros }\end{array}$ & $\begin{array}{l}\text { Fat people do not } \\
\text { necessarily eat more than } \\
\text { the others do }\end{array}$ \\
\hline
\end{tabular}


As respostas dos experts obtiveram 100\% de compreensão perfeita para 11 itens na equivalência semântica. Estes 11 itens foram, portanto, mantidos em sua primeira versão adaptada. Os demais 23 itens obtiveram graus variados de compreensão conforme apresentado na Tabela 1, na qual os valores mais elevados indicam maior grau de compreensão.

Uma professora de inglês foi consultada para discutir as dúvidas com relação aos itens que geraram compreensão insatisfatória e melhores traduções de alguns termos como a palavra sinful (em Being fat is sinful), e a expressão can't believe (em I can't believe someone of average weight would marry a fat person). Outros itens e expressões que suscitaram dúvidas nos comentários dos experts foram discutidos e revistos, gerando uma nova versão da escala traduzida. As melhores opções de resposta aos itens da escala foram também discutidas com os experts adotando-se as opções: discordo totalmente, discordo da maior parte, nem concordo nem discordo, concordo com a maior parte, e concordo totalmente.

As respostas para as versões inglês e português dos bilíngues foram comparadas com a finalidade de verificar a ocorrência de incongruências nas duas línguas como parte da equivalência semântica (Tabela 2).

O CCI em geral foi satisfatório com exceção dos itens $6,8,23$ e 31 . Tais resultados indicam que há baixa variabilidade intra-observador/bilíngue e maior variabilidade inter-observador/bilíngue, o que é esperado em um instrumento. Para os itens $2,6,7,17,29,30$ e 31 a correlação foi baixa $(<0,4)$, para as demais foi satisfatória $(0,4-0,75)$ ou excelente $(>0,75)$. Para os itens $2,7,17,30$ e 31, apesar da correlação ser baixa, o CCI é satisfatório $(>0,4)$ e não há diferença nas médias português-inglês. $\mathrm{O}$ item 21 foi respondido absolutamente da mesma maneira por todos os jo-

Tabela 1. Frequência de respostas dos experts aos itens com graus variados de compreensão da versão 1 traduzida para o português do Brasil do Antifat Attitudes Test $(\mathrm{n}=15)$.

\begin{tabular}{|c|c|c|c|c|c|}
\hline \multirow[b]{2}{*}{ Item } & \multicolumn{5}{|c|}{ Grau de compreensão } \\
\hline & $\begin{array}{c}1 \\
\text { n (\%) }\end{array}$ & $\begin{array}{c}2 \\
\text { n (\%) }\end{array}$ & $\begin{array}{c}3 \\
\text { n (\%) }\end{array}$ & $\begin{array}{c}4 \\
\text { n (\%) }\end{array}$ & $\begin{array}{c}5 \\
\text { n (\%) }\end{array}$ \\
\hline 2 & $0(0)$ & $1(6,7)$ & $0(0)$ & $0(0)$ & $14(93,3)$ \\
\hline 3 & $0(0)$ & $0(0)$ & $2(13,3)$ & $3(20,0)$ & $10(66,7)$ \\
\hline 5 & $0(0)$ & $0(0)$ & $0(0)$ & $1(6,7)$ & $14(93,3)$ \\
\hline 6 & $0(0)$ & $0(0)$ & $0(0)$ & $2(13,3)$ & $13(86,7)$ \\
\hline 7 & $0(0)$ & $0(0)$ & $1(6,7)$ & $0(0)$ & $14(93,3)$ \\
\hline 8 & $0(0)$ & $0(0)$ & $1(6,7)$ & $0(0)$ & $14(93,3)$ \\
\hline 11 & $0(0)$ & $0(0)$ & $1(6,7)$ & $1(6,7)$ & $13(86,7)$ \\
\hline 12 & $0(0)$ & $0(0)$ & $2(13,3)$ & $0(0)$ & $13(86,7)$ \\
\hline 13 & $0(0)$ & $0(0)$ & $0(0)$ & $1(6,7)$ & $14(93,3)$ \\
\hline 14 & $0(0)$ & $0(0)$ & $1(6,7)$ & $0(0)$ & $14(93,3)$ \\
\hline 15 & $0(0)$ & $0(0)$ & $0(0)$ & $1(6,7)$ & $14(93,3)$ \\
\hline 16 & $0(0)$ & $1(6,7)$ & $0(0)$ & $0(0)$ & $14(93,3)$ \\
\hline 18 & $0(0)$ & $0(0)$ & $0(0)$ & $1(6,7)$ & $14(93,3)$ \\
\hline 19 & $0(0)$ & $0(0)$ & $3(20,0)$ & $3(20,0)$ & $9(60,0)$ \\
\hline 20 & $0(0)$ & $0(0)$ & $1(6,7)$ & $0(0)$ & $14(93,3)$ \\
\hline 21 & $0(0)$ & $0(0)$ & $2(13,3)$ & $0(0)$ & $13(86,7)$ \\
\hline 23 & $0(0)$ & $0(0)$ & $1(6,7)$ & $2(13,3)$ & $12(80,0)$ \\
\hline 24 & $1(6,7)$ & $0(0)$ & $4(26,7)$ & $2(13,3)$ & $8(53,3)$ \\
\hline 25 & $0(0)$ & $0(0)$ & $1(6,7)$ & $1(6,7)$ & $13(86,7)$ \\
\hline 28 & $0(0)$ & $0(0)$ & $0(0)$ & $1(6,7)$ & $14(93,3)$ \\
\hline 29 & $0(0)$ & $0(0)$ & $1(6,7)$ & $1(6,7)$ & $13(86,7)$ \\
\hline 31 & $0(0)$ & $0(0)$ & $1(6,7)$ & $0(0)$ & $14(93,3)$ \\
\hline 32 & $0(0)$ & $0(0)$ & $0(0)$ & $1(6,7)$ & $14(93,3)$ \\
\hline
\end{tabular}

$1=$ não entendi nada/ 2 = entendi só um pouco/ 3 = entendi mais ou menos $/ 4=$ entendi quase tudo, mas tive algumas dúvidas/ 5 $=$ entendi perfeitamente e não tenho dúvidas. 
Tabela 2. Comparação das respostas dos bilíngues $(n=13)$ aos itens das versões do Antifat Attitudes Test em inglês e português do Brasil.

\begin{tabular}{|c|c|c|c|c|c|c|}
\hline Item & $\begin{array}{c}\text { Inglês } \\
\text { Média (dp) }\end{array}$ & $\begin{array}{l}\text { Português } \\
\text { Média (dp) }\end{array}$ & $\begin{array}{c}\text { Correlação } \\
(\mathbf{r})\end{array}$ & Valor-p & $\mathrm{CCI}^{\star}$ & $\begin{array}{l}\mathrm{X}^{2 * *} \\
\mathrm{Z}(\mathrm{p})\end{array}$ \\
\hline 1 & $\begin{array}{c}2,23 \\
(1,48)\end{array}$ & $\begin{array}{c}2,38 \\
(1,39)\end{array}$ & 0,799 & 0,001 & 0,891 & $-0,447(0,655)$ \\
\hline 2 & $\begin{array}{l}3,23 \\
(1,42)\end{array}$ & $\begin{array}{c}3,15 \\
(1,28)\end{array}$ & 0,395 & 0,181 & 0,510 & $-0,575(0,565)$ \\
\hline 3 & $\begin{array}{c}3,15 \\
(1,14)\end{array}$ & $\begin{array}{c}3,15 \\
(1,28)\end{array}$ & 0,576 & 0,040 & 0,708 & $-0,137(0,891)$ \\
\hline 4 & $\begin{array}{c}2,38 \\
(1,39)\end{array}$ & $\begin{array}{c}2,54 \\
(1,39)\end{array}$ & 0,629 & 0,021 & 0,730 & $<0,001(1,000)$ \\
\hline 5 & $\begin{array}{c}2,85 \\
(1,52)\end{array}$ & $\begin{array}{c}3,00 \\
(1,53)\end{array}$ & 0,820 & 0,001 & 0,905 & $-0,743(0,458)$ \\
\hline 6 & $\begin{array}{c}1,69 \\
(1,03)\end{array}$ & $\begin{array}{c}1,54 \\
(1,20)\end{array}$ & 0,232 & 0,446 & 0,143 & $-0,317(0,751)$ \\
\hline 7 & $\begin{array}{c}1,46 \\
(0,66)\end{array}$ & $\begin{array}{c}1,54 \\
(0,66)\end{array}$ & 0,366 & 0,219 & 0,692 & $-0,447(0,655)$ \\
\hline 8 & $\begin{array}{c}1,23 \\
(0,60)\end{array}$ & $\begin{array}{c}1,62 \\
(1,33)\end{array}$ & 0,685 & 0,010 & 0,398 & $-1,069(0,285)$ \\
\hline 9 & $\begin{array}{c}1,23 \\
(0,83)\end{array}$ & $\begin{array}{c}1,31 \\
(0,86)\end{array}$ & 0,736 & 0,004 & 0,972 & $-1,000(0,317)$ \\
\hline 10 & $\begin{array}{c}1,31 \\
(0,48)\end{array}$ & $\begin{array}{c}1,54 \\
(0,97)\end{array}$ & 0,981 & $<0,001$ & 0,818 & $-1,342(0,180)$ \\
\hline 11 & $\begin{array}{c}2,31 \\
(1,25)\end{array}$ & $\begin{array}{c}2,00 \\
(1,08)\end{array}$ & 0,610 & 0,027 & 0,599 & $-0,743(0,458)$ \\
\hline 12 & $\begin{array}{c}1,54 \\
(0,66)\end{array}$ & $\begin{array}{c}1,38 \\
(0,65)\end{array}$ & 0,730 & 0,005 & 0,782 & $-1,000(0,317)$ \\
\hline 13 & $\begin{array}{c}2,23 \\
(1,30)\end{array}$ & $\begin{array}{c}1,85 \\
(1,14)\end{array}$ & 0,584 & 0,036 & 0,818 & $1,406(0,160)$ \\
\hline 14 & $\begin{array}{c}4,15 \\
(1,21)\end{array}$ & $\begin{array}{c}4,46 \\
(1,13)\end{array}$ & 0,645 & 0,017 & 0,539 & $-0,736(0,461)$ \\
\hline 15 & $\begin{array}{c}3,08 \\
(1,38)\end{array}$ & $\begin{array}{c}2,69 \\
(1,70)\end{array}$ & 0,758 & 0,003 & 0,826 & $-1,127(0,260)$ \\
\hline 16 & $\begin{array}{c}1,15 \\
(0,38)\end{array}$ & $\begin{array}{c}1,15 \\
(0,56)\end{array}$ & 0,677 & 0,011 & 0,772 & $<0,001(1,000)$ \\
\hline 17 & $\begin{array}{c}2,08 \\
(1,12)\end{array}$ & $\begin{array}{c}1,31 \\
(0,63)\end{array}$ & 0,369 & 0,215 & 0,545 & $-2,232(0,026)$ \\
\hline 18 & $\begin{array}{c}2,31 \\
(1,32)\end{array}$ & $\begin{array}{c}1,92 \\
(1,19)\end{array}$ & 0,796 & 0,001 & 0,896 & $-1,633(0,102)$ \\
\hline 19 & $\begin{array}{c}1,46 \\
(0,88)\end{array}$ & $\begin{array}{c}1,54 \\
(1,20)\end{array}$ & 0,549 & 0,052 & 0,897 & $-0,447(0,655)$ \\
\hline 20 & $\begin{array}{c}1,69 \\
(0,75)\end{array}$ & $\begin{array}{c}1,69 \\
(1,18)\end{array}$ & 0,830 & $<0,001$ & 0,854 & $<0,001(1,000)$ \\
\hline 21 & $\begin{array}{c}1,00 \\
(0,00)\end{array}$ & $\begin{array}{c}1,00 \\
(0,00)\end{array}$ & 1,000 & - & - & $<0,001(1,000)$ \\
\hline 22 & $\begin{array}{c}1,69 \\
(0,86)\end{array}$ & $\begin{array}{c}1,46 \\
(0,78)\end{array}$ & 0,520 & 0,069 & 0,649 & $-1,000(0,317)$ \\
\hline 23 & $\begin{array}{c}4,38 \\
(0,87) \\
\end{array}$ & $\begin{array}{c}4,38 \\
(1,04) \\
\end{array}$ & 0,416 & 0,158 & 0,435 & $-0,137(0,891)$ \\
\hline
\end{tabular}


Tabela 2. continuação

\begin{tabular}{|c|c|c|c|c|c|c|}
\hline Item & $\begin{array}{c}\text { Inglês } \\
\text { Média (dp) }\end{array}$ & $\begin{array}{l}\text { Português } \\
\text { Média (dp) }\end{array}$ & $\begin{array}{c}\text { Correlação } \\
(\mathbf{r})\end{array}$ & Valor-p & $\mathrm{CCI}^{\star}$ & $\begin{array}{l}\mathrm{X}^{2 \star *} \\
\mathrm{Z}(\mathrm{p})\end{array}$ \\
\hline 24 & $\begin{array}{c}1,92 \\
(1,19)\end{array}$ & $\begin{array}{c}2,23 \\
(1,17)\end{array}$ & 0,596 & 0,032 & 0,714 &,$- 0962(0,336)$ \\
\hline 25 & $\begin{array}{c}2,00 \\
(1,08)\end{array}$ & $\begin{array}{c}1,77 \\
(1,17)\end{array}$ & 0,649 & 0,016 & 0,841 & $-1,000(0,317)$ \\
\hline 26 & $\begin{array}{c}2,08 \\
(1,04)\end{array}$ & $\begin{array}{c}1,54 \\
(0,66)\end{array}$ & 0,847 & $<0,001$ & 0,832 & $-2,333(0,020)$ \\
\hline 27 & $\begin{array}{c}2,00 \\
(1,16)\end{array}$ & $\begin{array}{c}2,15 \\
(1,52)\end{array}$ & 0,630 & 0,021 & 0,814 & $-0,513(0,608)$ \\
\hline 28 & $\begin{array}{c}2,08 \\
(1,26)\end{array}$ & $\begin{array}{c}2,08 \\
(1,26)\end{array}$ & 0,601 & 0,030 & 0,732 & $-0,087(0,931)$ \\
\hline 29 & $\begin{array}{c}3,15 \\
(1,14)\end{array}$ & $\begin{array}{c}3,77 \\
(1,24)\end{array}$ & 0,043 & 0,888 & $-0,199$ & $-1,205(0,228)$ \\
\hline 30 & $\begin{array}{c}1,54 \\
(0,97)\end{array}$ & $\begin{array}{c}1,23 \\
(0,60)\end{array}$ & 0,321 & 0,285 & 0,722 & $-1,414(0,157)$ \\
\hline 31 & $\begin{array}{c}3,69 \\
(1,38)\end{array}$ & $\begin{array}{c}3,31 \\
(1,60)\end{array}$ & 0,290 & 0,337 & 0,425 & $-0,768(0,443)$ \\
\hline 32 & $\begin{array}{c}3,15 \\
(1,35)\end{array}$ & $\begin{array}{c}3,15 \\
(1,41)\end{array}$ & 0,672 & 0,012 & 0,818 & $-0,333(0,739)$ \\
\hline 33 & $\begin{array}{c}1,62 \\
(0,87)\end{array}$ & $\begin{array}{c}1,31 \\
(0,63)\end{array}$ & 0,707 & 0,007 & 0,792 & $-1,633(0,102)$ \\
\hline 34 & $\begin{array}{c}3,62 \\
(1,39)\end{array}$ & $\begin{array}{c}3,85 \\
(1,57)\end{array}$ & 0,735 & 0,004 & 0,936 & $-1,134(0,257)$ \\
\hline
\end{tabular}

${ }^{\star}$ CCI: coeficiente de correlação intraclasse. ${ }^{* *} \mathrm{X}^{2}$ : Wilcoxon (no SPSS os ranks negativos do teste Wilcoxon são convertidos em Z escore).

vens bilíngues, tanto em português como inglês, com variância zero. Portanto, não há valor de $\mathrm{p}$ ou CCI.

O teste de Wilcoxon apontou diferença na média das respostas para os itens 17 e 26, que tiveram as respostas analisadas individualmente, com reenvio para os bilíngues (juntamente com outras de maior incongruência) com a seguinte pergunta: Você realmente entende de forma diferente o mesmo item em português e em inglês ( $f a-$ vor explicar os motivos) ou acredita que pode ter havido algum engano durante o preenchimento?

Doze dos treze bilíngues responderam essencialmente que "erraram o preenchimento" ou "que mudaram a forma de pensar quando responderam a escala pela segunda vez". Pode-se conjecturar, em função destas respostas, que estes bilíngues não responderam o teste com a atenção necessária nas duas línguas, mas também que o AFAT contém afirmações que suscitam a reflexão do indivíduo sobre a questão, com a possibilidade de mudança de opinião quando se pensa novamente sobre a pergunta.
Os comentários dos bilíngues com relação à tradução e termos foram considerados e se realizou nova consulta aos experts sobre diferença na interpretação dos itens em português e em inglês gerando nova versão do instrumento. Os experts concordaram com a tradução sugerida para a maior parte dos itens, e algumas sugestões de alteração pertinentes foram realizadas gerando a versão final adaptada (Quadro 1).

Com relação às opções de resposta à escala, os cinco pontos traduzidos literalmente do inglês listados anteriormente, foram rediscutidos com os experts após aplicação do questionário na equivalência operacional. Desta forma, optou-se por alterar a palavra "discordo" por "não concordo", uma vez que se trata de uma expressão de uso mais corriqueiro na língua portuguesa. Assim, o formato adotado para aplicação da escala foi o de tabela com opções de resposta em escala Likert de cinco pontos: "Não concordo com nada", "não concordo com a maior parte", "nem discordo nem concordo", "concordo com a maior parte" e "concordo totalmente". 
Ainda na equivalência operacional, (aplicação da versão traduzida para estudantes de $\mathrm{Nu}$ trição e Saúde Pública $-\mathrm{n}=37$ ), uma minoria referiu alguma "dificuldade": dois referiram dificuldade no preenchimento da escala; dois não consideraram as opções de resposta claras; e um considerou os itens repetitivos. Nove estudantes (24,3\%) questionaram a utilização da palavra "gordo", sinalizando que a palavra traz uma conotação negativa para a pessoa obesa. $\mathrm{O}$ tempo de preenchimento da escala foi de cinco a dez minutos.

Após estas etapas, a versão final da AFAT foi retrotraduzida, a tradução e a retrotradução foram discutidas pelos autores deste trabalho e discrepâncias não foram encontradas.

Os estudantes que participaram da análise de mensuração $(\mathrm{n}=340)$ tinham em média 25,8 anos (DP 7,64); 81,4\% eram do sexo feminino $(\mathrm{N}=276)$ e possuíam Índice de Massa Corpórea (IMC) médio de $24,39 \mathrm{~kg} / \mathrm{m}^{2}$ (DP 4,67). A pontuação na AFAT variou de 35 a 117, com média de 63,69 (DP 14,74). Considerando a forma de pontuação recomendada pelos autores do original (escore total dos 34 itens dividido por 34), a média foi de 1,87 (DP 0,43). A consistência interna ( $\alpha$ Cronbach) da AFAT global foi de 0,85 . Para as mulheres, foi de 0,82 e 0,86 , para os homens - Tabela 3.

Do total de estudantes desta etapa, 76 responderam ao reteste $(22,35 \%)$. A pontuação média no reteste foi de 1,73 (DP 0,49) e o CCI entre as medidas foi de 0,83 .

A análise fatorial confirmatória apresentou um Comparative Fit Index (CFI) de 0,931; Tucker-Lewis Index (TLI) de 0,926 e Root Mean Square Error of Aproximation (RMSEA) de 0,068, o que indica boa qualidade de ajuste. Ou seja, é possível manter as subescalas encontradas na escala origi- nal. Além disso, as covariâncias entre as subescalas revelaram elevada correlação $(0,785$ entre as subescalas" controle do peso e culpa" e "depreciação social e do caráter"; 0,803 entre as subescalas "controle do peso e culpa" e "não atratividade física e romântica"; e 0,862 entre as subescalas "não atratividade física e romântica" e "depreciação social e do caráter"), conforme representado nas setas entre as subescalas na Figura 1.

As cargas fatoriais relativas aos itens que compõem as subescalas também estão apresentadas na Figura 1. As informações referentes aos itens com sentido diferente das demais (invertidas para o cálculo do escore) apresentam o sinal negativo.

Os escores e respectivas consistências internas das subescalas, após aplicação nos estudantes (N =340) estão também apresentados na Tabela 3.

\section{Discussão}

O processo de adaptação transcultural da AFAT, originalmente desenvolvida nos Estados Unidos em inglês para o português do Brasil, foi realizado segundo as recomendações ${ }^{33}$, encontrando boa equivalência em todas as etapas.

Após a discussão da versão traduzida em português com os experts e os bilíngues, foram realizadas alterações em oito dos 34 itens. Estas ocorreram devido à tradução inicial de palavras em inglês para outras em português, que não correspondiam à palavra - ou expressão mais empregada na língua Portuguesa; como as expressões "me associar", "pouco atrativas" e "relacionamento romântico". Outras alterações foram realizadas com o objetivo de melhorar a compreensão do item relacionando-a ao indivíduo obeso e não a alguém com sobrepeso, como aconteceu na troca

Tabela 3. Pontuação média e consistência interna ( $\alpha$ de Cronbach) da escala global e das subescalas da versão em português do Brasil do Antifat Attitudes Test $(\mathrm{n}=340)$.

\begin{tabular}{|c|c|c|c|c|}
\hline $\begin{array}{c}\text { Escala global ou } \\
\text { Subescala }\end{array}$ & $\begin{array}{l}\text { Número de } \\
\text { afirmações }\end{array}$ & Afirmações & $\begin{array}{l}\text { Pontuação média } \\
\text { (DP) }\end{array}$ & $\alpha$ de Cronbach \\
\hline Escala global & 34 & $1-34$ & $\begin{array}{c}1,87 \\
(0,43)\end{array}$ & $\begin{array}{c}0,85 \\
(0,82 \text { mulheres; } 0,86 \text { homens })\end{array}$ \\
\hline $\begin{array}{l}\text { Depreciação } \\
\text { social e do caráter }\end{array}$ & 15 & $\begin{array}{c}6,7,8,9,11,12,14 \\
16,19,20,21,22,23 \\
30,33\end{array}$ & $\begin{array}{c}1,40 \\
(0,38)\end{array}$ & $\begin{array}{c}0,72 \\
(0,66 \text { mulheres; } 0,79 \text { homens })\end{array}$ \\
\hline $\begin{array}{l}\text { Não atratividade } \\
\text { física e romântica }\end{array}$ & 10 & $\begin{array}{c}2,4,5,10,17,24,26 \\
27,29,31\end{array}$ & $\begin{array}{c}2,01 \\
(0,63)\end{array}$ & $\begin{array}{c}0,62 \\
(0,61 \text { mulheres; } 0,63 \text { homens })\end{array}$ \\
\hline $\begin{array}{l}\text { Controle do peso } \\
\text { e culpa }\end{array}$ & 9 & $\begin{array}{c}1,3,13,15,18,25,28 \\
32,34\end{array}$ & $\begin{array}{c}2,52 \\
(0,67) \\
\end{array}$ & $\begin{array}{c}0,70 \\
(0,69 \text { mulheres; } 0,70 \text { homens }) \\
\end{array}$ \\
\hline
\end{tabular}




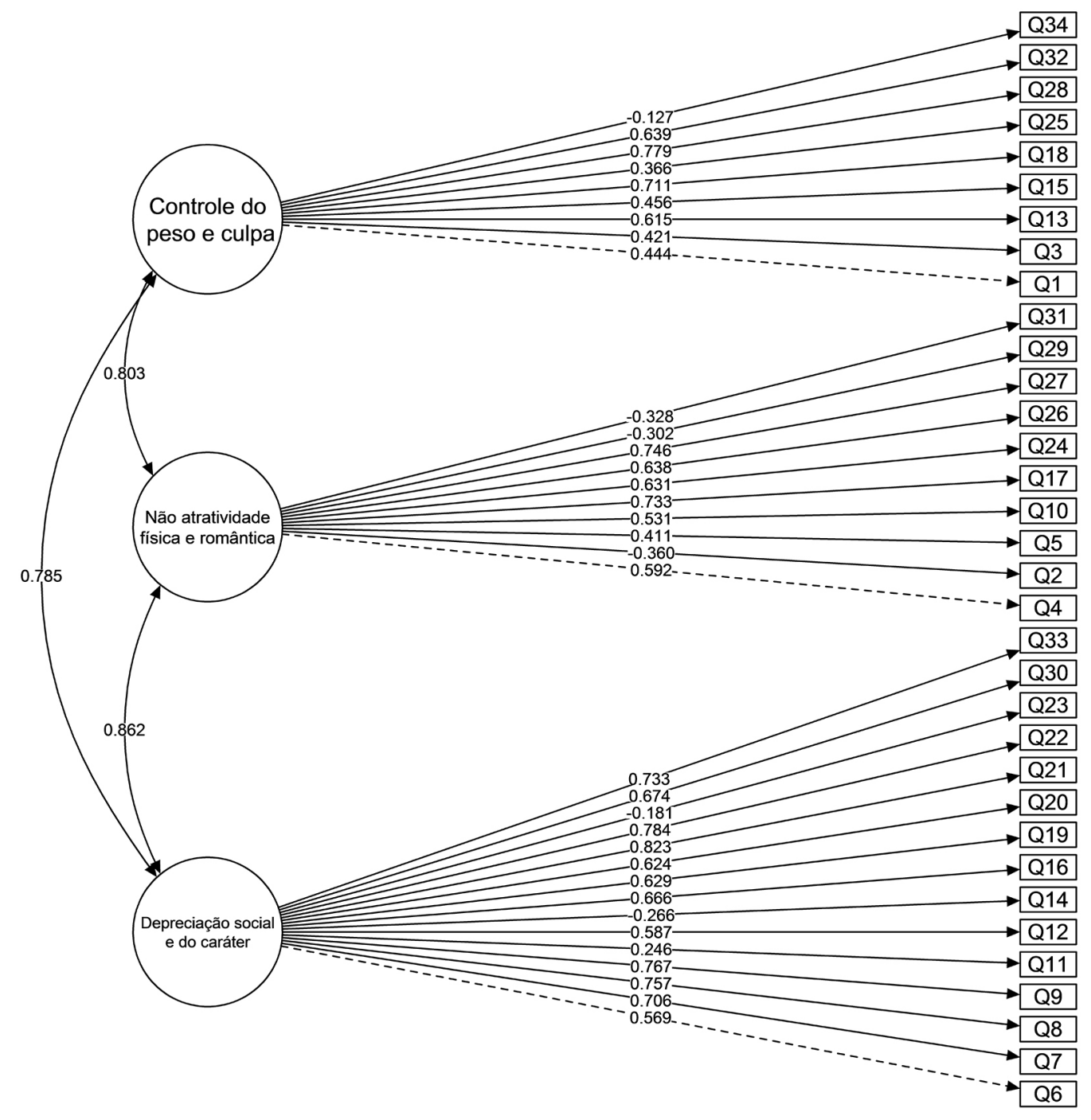

Figura 1. Subescalas e cargas fatoriais obtidas na análise fatorial confirmatórias dos itens da versão final do Antifat Attitudes Test $(\mathrm{n}=340)$.

da expressão "começasse a engordar" por "começasse a ficar gordo (a)" e "tornasse gordo (a)". Tais alterações são esperadas no processo de adaptação transcultural e permitem que o instrumento alcance o melhor entendimento possível na língua em que será empregado.

O pré-teste com os estudantes de graduação e pós-graduação resultou em reduzida dificuldade no preenchimento, mas indicou a necessidade de alterar a escala de respostas para facilitar a compreensão das opções (ex: "discordo" por "não concordo"). Com relação à indicação de que os itens seriam repetitivos, entende-se que a AFAT possui muitas afirmações negativas relacionadas aos indivíduos percebidos como "gordos" ou obesos, mas não há itens com sentidos idênticos. E itens semelhantes - às vezes com sentido de resposta invertido - são uma estratégia da criação de escalas para garantir respostas não aleatórias. Com relação ao questionamento da utilização da 
palavra "gordo" e da expressão "pessoas gordas", o objetivo da escala é avaliar as reações dos participantes em relação ao indivíduo percebido como gordo (inclusive dos estereótipos e pensamentos associados aos termos). Na versão em inglês utiliza-se o termo fat people e não obese person; assim os termos foram mantidos.

A análise de equivalência semântica realizada com os jovens bilíngues demonstrou a necessidade de revisar os itens com a presença de incongruências (baixa correlação intraclasse); no entanto, quando questionados em relação às afirmações apontadas, observou-se que as diferenças nas respostas ocorreram em decorrência de falta de atenção durante o preenchimento - relatada pelos participantes.

A consistência interna da AFAT adaptada é satisfatória; para a escala total e duas das subescalas ela foi boa, no entanto para subescala "não atratividade física e romântica" a consistência foi menor, mas satisfatória. A mesma análise conduzida na escala original ${ }^{32}$ encontrou $\alpha$ de Cronbach de 0,95 para a escala total para cada um dos sexos.

Comparando-se a consistência interna $(\alpha$ Cronbach) das subescalas com as da versão original (a saber: 0,91 para homens e 0,87 para mulheres na subescala $1 ; 0,79$ para homens e 0,84 para mulheres na subescala 2; e 0,77 para homens e 0,85 para mulheres na subescala 3 ) pode-se observar que os valores foram menores para a versão adaptada - mas todos bons ou satisfatórios - evidenciando-se ainda a tendência de valores maiores para os homens na subescala 1.

A análise fatorial confirmatória apresentou índices de qualidade de ajuste (CFI, TLI e RMSEA) adequados, o que demonstra que as subescalas da escala original podem ser mantidas na adaptação realizada neste estudo, não sendo necessária nova análise exploratória.
A confiabilidade teste-reteste da escala também foi satisfatória. No estudo de desenvolvimento da AFAT esta análise não foi realizada, mas os autores destacaram a necessidade de avaliar a estabilidade temporal da escala ${ }^{32}$ - que foi atestada pelos resultados desta validação. Com relação à percepção pelas respostas dos bilíngues de que os itens da AFAT suscitam certa reflexão sobre a questão "antiobesidade" (com a possibilidade de mudança de opinião quando se pensa novamente sobre a pergunta); o resultado da confiabilidade teste-reteste encontrado aponta que, mesmo havendo esta reflexão e eventual mudança de opinião, a escala se manteve estável entre o período aqui analisado. Novas avaliações temporais, no entanto, podem ser conduzidas para melhor entendimento desta questão.

São limitações deste estudo a maior presença de mulheres na amostra para avaliação da validade (análise de mensuração) e o fato de o estudo ser conduzido com uma população específica (estudantes de graduação da área da saúde), dificultando a generalização dos resultados e aplicação da escala em populações muito diferentes da que participou do processo de adaptação. De qualquer forma, como colocado na introdução, um dos públicos de interesse para estudo de atitudes negativas com relação aos obesos é justamente estudantes e profissionais da área da saúde.

Considerando os achados, conclui-se que o processo de adaptação transcultural foi adequado e a escala AFAT apresentou propriedades psicométricas satisfatórias; sendo assim, pode ser considerado um instrumento útil para avaliação da presença de atitudes negativas em relação à obesidade e aos obesos. Uma vez que vários estudos apontam que estas atitudes são prevalentes, é importante avaliá-las com instrumentos adequados e válidos para o planejamento de intervenções educativas sobre o tema. 


\section{Colaboradores}

AA Obara e MS Alvarenga participaram igualmente de todas as etapas de elaboração do artigo.

\section{Agradecimentos}

Agradecemos aos profissionais e estudantes envolvidos no processo de adaptação transcultural.

\section{Referências}

1. World Health Organization (WHO). Global status report on noncommunicable diseases. Geneva: WHO; 2010.

2. Brewis AA. Stigma and the perpetuation of obesity. Soc Sci Med 2014; 118:152-158.

3. Budd GM, Mariotii M, Graff D, Falkenstein K. Health care professionals' attitudes about obesity: an integrative review. Appl Nurs Res 2011; 24(3):127-137.

4. Swinburn BA, Sacks G, Hall KD, McPherson K, Finegood DT, Moodie MJ, Gortmaker SL. The global obesity pandemic: shaped by global drivers and local environments. Lancet 2011; 378(9793):804-814.

5. Puhl RM, Brownell KD. Bias, discrimination and obesity. Obes Res 2001; 9(12):788-805.

6. Puhl RM, Heuer CA. The stigma of obesity: a review and update. Obesity 2009; 17(5):941-964.

7. Puhl RM, Heuer CA. Obesity stigma: important considerations for Public Health. Am J Public Health 2010; 100(6):1019-1028.

8. Harvey EL, Hill AJ. Health professionals' views of overweight people and smokers. Int J Obes Relat Metab Disord 2001; 25(8):1253-1261.

9. Teachman BA, Brownell KD. Implicit anti-fat bias among health professionals: is anyone immune? Int $J$ Obes Relat Metab Disord 2001; 25(10):1525-1531.

10. Brown I. Nurses' attitudes towards adult patients who are obese: literature review. J Adv Nurs 2006; 53(2):221232.

11. Poon M-Y, Tarrant M. Obesity: attitudes of undergraduate student nurses and registered nurses. J Clin Nurs 2009; 18(16):2355-2365.

12. Sabin JA, Marini M, Nosek BA. Implicit and explicit anti-fat bias among a large sample of medical doctors by BMI, race/ethnicity and gender. PLoS One 2012; 7(11):e48448.

13. Pantenburg B, Sikorski C, Luppa M, Schomerus G, Konig HH, Werner P, Riedel-Heller SG. Medical students' attitudes towards overweight and obesity. PLoS One 2012; 7(11):e48113.

14. Phelan SM, Dovidio JF, Puhl RM, Burgess DJ, Nelson DB, Yeazel MW, Hardeman R, Perry S, van Ryn M. Implicit and explicit weight bias in a national sample of 4,732 medical students: the Medical Student CHANGES Study. Obesity 2014; 22(4):1201-1208.

15. Robinson EL, Ball LE, Leveritt MD. Obesity bias among health and non-health students attending an Australian university and their perceived obesity education. $J$ Nutr Educ Behav 2014; 46(5):390-395.

16. Goffman E. Stigma: notes on the management of spoiled identity. New York: Symon and Schuster Inc; 1963.

17. Monteiro S, Villela W. Estigma e saúde. Rio de Janeiro: Fiocruz, 2013.

18. Daníelsdóttir S, O’Brien KS, Ciaoc A. Anti-fat prejudice reduction: a review of published studies. Obes Facts 2010; 3(1):47-58.

19. Andreyeva T, Puhl RM, Brownell KD. Changes in perceived weight discrimination among Americans, $1995-$ 1996 through 2004-2006. Obesity 2008; 16(5):11291134. 
20. Eagly AH, Chaiken S. The psychology of attitudes. Orlando: Harcourt Brace Jovanovich College Publishers; 1993.

21. Fishbein M, Ajzen I. Belief, attitude, intention and behavior: an introduction to theory and research. Massachusetts: Addison-Wesley; 1975.

22. Schwartz MB, Vartanian LR, Nosek BA, Brownell KD. The influence of one's own body weight on implicit and explicit anti-fat bias. Obesity 2006; 14(3):440-447.

23. Brownell KD, Puhl RM, Schwartz MB, Rudd L. Weight bias: nature, consequences, and remedies. New York: The Guildford Press; 2005.

24. Allison DB, Basile VC, Yuker HE. The measurement of attitudes toward and beliefs about obese persons. Int J Eat Disord 1991; 10(5):599-607.

25. Watson L, Oberle K, Deutscher D. Development and psychometric testing of the Nurses' Attitudes Toward Obesity and Obese Patients (NATOOPS) Scale. Res Nurs Health 2008; 31(6):586-593.

26. Ip EH, Marshall S, Vitolins M, Crandall SJ, Davis S, Miller D, Kronner D, Vaden K, Spangler J. Measuring medical student attitudes and beliefs regarding patients who are obese. Acad Med 2013; 88(2):282-289.

27. Bacon JG, Scheltema KE, Robinson BE. Fat phobia scale revisited: the short form. Int J Obes 2001; 25(2):252257.

28. Puhl RM, Schwartz MB, Brownell KD. Impact of perceived consensus on stereotypes about obese people: a new approach for reducing bias. Health Psychol 2005; 24(5):517-525.

29. Cori GC, Petty MLB, Alvarenga MS. Atitudes de nutricionistas em relação a indivíduos obesos - um estudo exploratório. Cien Saude Colet 2015; 20(2):9-20.

30. Teixeira FV, Pais-Ribeiro JL, Maia ARP da C. Crenças e práticas dos profissionais de saúde face à obesidade: uma revisão sistemática. Rev Assoc Med Bras 2012; 58(2):254-262.

31. Grejanin DKM, Pezzo TH, Nastri V, Sanches VPP, Nascimento DDG, Quevedo MP. As percepções sobre o "ser obeso" sob a ótica do paciente e dos profissionais da saúde. Rev Bras Crescimento Desenvolv Hum 2007; 17(3):37-47.

32. Lewis RJ, Cash TF, Jacobi L, Bubb-Lewis C. Prejudice toward fat people: The development and validation of the Antifat Attitudes Test. Obes Res 1997; 5(4):297-307.

33. Reichenheim ME, Moraes CL. Operacionalização de adaptação transcultural de instrumentos de aferição usados em epidemiologia. Rev Saude Publica 2007; 41(4):665-673.

Artigo apresentado em 05/03/2016

Aprovado em 01/08/2016

Versão final apresentada em 03/08/2016 